\title{
Risk Factors Associated with Group B Streptococcus Colonization and Their Effect on Pregnancy Outcome
}

\author{
Lucia Matsiane Lekala ${ }^{1}$, Rooyen Tinago Mavenyengwa ${ }^{2,3}$, *, Sylvester Rogers Moyo ${ }^{3}$, \\ Sogolo Lucky Lebelo ${ }^{4}$, John Yenga Bolukaoto ${ }^{4}$, Martina Onyedi Chukwu ${ }^{4}$, \\ Charles Maropeng Monyama ${ }^{4}$, Maphoshane Nchabeleng ${ }^{5}$, Tshimane Charles Tshepuwane ${ }^{1}$, \\ Sam Monokoane ${ }^{1}$ \\ ${ }^{1}$ Department of Obstetrics and Gynaecology, Sefako Makgatho Health Sciences University, Pretoria, South Africa \\ ${ }^{2}$ Department of Medical Microbiology, University of Zimbabwe College of Health Sciences, Harare, Zimbabwe \\ ${ }^{3}$ Department of Health Sciences, Faculty of Health and Applied Sciences, Namibia University of Science and Technology, Windhoek, \\ Namibia \\ ${ }^{4}$ Department of Life and Consumer Sciences, College of Agriculture \& Environmental Science, University of South Africa, Pretoria, South \\ Africa \\ ${ }^{5}$ Department of Microbiological Pathology, Sefako Makgatho Health Sciences University, Pretoria, South Africa
}

\section{Email address:}

sebotselekala@yahoo.com (L. M. Lekala),rmavenyengwa@yahoo.com (R. T. Mavenyengwa), srmoyo@polytechnic.edu.na (S. R. Moyo), lebelol@unisa.ac.za (S. L. Lebelo), jbolukaoto@gmail.com (J. Y. Bolukaoto), timummy2002@yahoo.com (M. O. Chukwu), mcmonyama@gmail.com (C. M. Monyama),maphoshane.nchabeleng@smu.ac.za (M. Nchabeleng), Sam.Monokoane@ul.ac.za (S. Monokoane),charles4@yebo.co.za (T. C. Tshepuwane)

To cite this article:

Lucia Matsiane Lekala, Rooyen Tinago Mavenyengwa, Sylvester Rogers Moyo, Sogolo Lucky Lebelo, John Yenga Bolukaoto, Martina Onyedi Chukwu, Charles Maropeng Monyama, Maphoshane Nchabeleng, Tshimane Charles Tshepuwane, Sam Monokoane. Risk Factors Associated with Group B Streptococcus Colonization and Their Effect on Pregnancy Outcome. Journal of Gynecology and Obstetrics. Vol. 3, No. 6, 2015, pp. 121-128. doi: 10.11648/j.jgo.20150306.14

\begin{abstract}
Background: Group B Streptococcus (GBS) is an important cause of maternal and neonatal morbidity and mortality in many parts of the world. It has been implicated in adverse pregnancy outcomes. Maternal colonization has been found to be a major risk factor for invasive neonatal GBS disease. The main objective of this study was to identify the risk factors of Group B streptococcus colonization and its effect on pregnancy outcome. Methods: This was a prospective study in which pregnant women attending antenatal clinic (ANC) at Dr George Mukhari Hospital (DGMH) were recruited. These were at least 18 years old and were at 16 weeks gestation. Vaginal and rectal swabs were taken at recruitment. Follow up of mothers until six weeks post-delivery and babies until three months of age was done. Results: A total of 340 pregnant women were then investigated for GBS colonization. Out of this number, $164(48.2 \%)$ were GBS positive. The majority of the women (62.1\%) were parity 1 and 2. Group B Streptococcus colonization was significant in women who had no matric education and who were unemployed. There was a significantly higher GBS colonization in women with previous history of miscarriages and stillbirths. The proportion of HIV positive, GBS colonized women was significant at $41.5 \%$ as compared to HIV negative GBS colonized (34.7\%). Eight women (4.9\%) presented with premature rupture of membranes (PROM) and the duration ranged from 0.5 to 72 hours. Ten $(6.1 \%)$ women had preterm deliveries. Three $(1.8 \%)$ women had wound sepsis post caesarean section and one $(0.6 \%)$ had endometritis post vaginal delivery. A total of $7(24.1 \%)$ babies were GBS positive. The number of live babies delivered was 167, with 1 case of miscarriage due to severe preeclampsia. There were 2 cases of early neonatal deaths which included 1 case of sepsis whereas the other one had multiple congenital abnormalities. Conclusion: Colonization by GBS in pregnant women at DGMH was high. Maternal risk factors identified were previous history of stillbirths/miscarriages, lack of education and being HIV positive. Overall pregnancy outcome both maternal and foetal was good.
\end{abstract}

Keywords: Group B Streptococcus, Risk Factors, Pregnancy, HIV, South Africa 


\section{Introduction}

Group B streptococcus (GBS), also known as Streptococcus agalactiae is an important cause of maternal and neonatal morbidity and mortality in many parts of the world [1]. It is also implicated in adverse pregnancy outcomes [2]. Maternal colonization has been found to be a major risk factor for invasive neonatal GBS disease within 6 days of birth [3]. In more than $80 \%$ of these cases, neonatal GBS infection is acquired during pregnancy and delivery by direct mother to child transmission of the pathogen. A study done in South Africa indicated a huge burden of GBS infection, in infants with early and late onset diseases suggesting that it is an important public health problem in South Africa [4]. The role of GBS in poor pregnancy outcome in the South African population is still unknown [5]. Epidemiologic studies showing the full extent of GBS colonization effect on pregnancy in the country are needed. There is no protocol for screening for GBS or a policy for GBS prophylaxis in pregnancy in South Africa.

Group B streptococcus has been isolated from genital or lower gastrointestinal tract of women at rates ranging from $5-40 \%$ [6]. It has been implicated as a cause of stillbirths [7]. Estimation of GBS colonization rates amongst women during pregnancy and determining its effect on pregnancy can contribute to prevention of negative pregnancy outcomes. An early study done in Zimbabwe showed a GBS colonization rate of $32 \%$ amongst pregnant women [7]. This indicated a potentially serious situation in the Southern African region. Previous studies on maternal factors associated with neonatal GBS disease have reported black race, history of previous miscarriage and stillbirths, age of less than 20 years and pre-term delivery as risk factors [8]. Group B Streptococcus is identified as a cause of pneumonia, sepsis and meningitis in neonates and of puerperal sepsis, amnionitis and endometritis in adults [6]. Poor maternal socio-economic status has been implicated as one of the risk factors for GBS infection [9]. Colonization rates vary among ethnic groups, geographic locations and age but rates are similar for pregnant and non-pregnant women [10].

The influence of maternal urogenital carriage of GBS on pregnancy and delivery outcomes has not been widely studied in South Africa. Elsewhere studies have shown the association of GBS with intrauterine infection [11, 12]. A study in Malawi compared the correlation of GBS and HIV, but found no overall difference between HIV status and GBS carriage women [13]. Group B streptococcus infection though, remains the leading cause of infectious mortality and morbidity among new-borns. The main objective of the current study was to establish risk factors for colonization and determine the effect of GBS colonization on pregnancy outcome at DGMH.

\section{Material and Methodology}

\subsection{Study Design}

This was a descriptive, prospective study, where pregnant women presenting to the antenatal clinic (ANC) from 16 weeks were recruited to form a cohort. The study was done from 15 February 2012 to 18 July 2012 and GBS positive women were followed-up until February 2013. The follow-up was done to collect the repeat sample when they presented in labour. Post-delivery women were followed up until six weeks for sepsis and babies until 3 months of age. A research nurse assisted in the follow-up of the cohort group.

\subsection{Study Population}

Pregnant women attending antenatal care clinic at $\mathrm{Dr}$ George Mukhari Hospital (DGMH) from 16 week gestation were recruited to form a cohort. This included 3 sectors of the South African population namely urban, urban-rural, and typical rural who were black Africans of different ethnicities.

\subsection{Sample Size}

Sample size calculation was done based on a prevalence of Streptococcus agalactiae in the Southern African region. Annual delivery rate at DGMH is 9000 births and the expected deliveries during one study are approximately 900 per year. At an expected frequency of $10 \%$, confidence level of $95 \%$ and a margin of error of 5\% sample size will be 185 . Due to the challenges of retention which are expected with a cohort, a sample size was increased to 350 to accommodate for those who will be lost to follow-up and home deliveries. The sample size has been calculated to have more than $90 \%$ power. Loss to follow-up was accommodated by increasing to 350 , which is slightly above the calculated sample size.

\subsection{Inclusion and Exclusion Criteria}

The inclusion criterion was recruitment of pregnant women attending ANC at DGMH from 16 weeks gestation to form the cohort aged 18 years and above. The women who were excluded were those who had any illness which required hospitalization by the attending physician and those who had received antibiotics 2 weeks prior to recruitment into the study.

\subsection{Data Collection Tool}

A questionnaire was used to collect the socio-demographic data from the participants. Each recruited woman was interviewed and socio-demographic characteristics which included gender, age, and marital status, residence, previous, current, own, rented or lodging. Information regarding obstetric history: previous miscarriages, previous stillbirth, previous deliveries was also collected. Information on the history of current pregnancy which included pyrexic illness in early pregnancy and vaginal discharge was also collected. 


\subsection{Specimen Collection and Analysis}

Vaginal and rectal swabs were collected and transported in Amies' transport media (Rochelle Chemicals and Lab Equipment, Pretoria, South Africa) to the Sefako Makgatho Health Sciences University Microbiology Laboratory for analysis. Another set of swabs were also collected at labour. No antiseptic preparation on the perineum or vulva was used before swabbing. Post-delivery women were followed up until six weeks for any sign of sepsis. The swabs were inoculated onto $5 \%$ blood agar with $8 \mathrm{ug} / \mathrm{ml}$ of gentamycin and $15 \mathrm{ug} / \mathrm{ml}$ of nalidixic acid and also into selective broth medium consisting of Todd Hewitt broth with the same antibiotics. After 24 hours incubation at $37^{\circ} \mathrm{C}$, blood agar plates were inspected for suspicious haemolytic and nonhaemolytic colonies. Suspicious colonies were processed using standard routine microbiological which included the CAMP test and confirmed using the latex agglutination test kit (Streptex - Slidex, Bio-Merieux, France).

\subsection{Data Analysis}

Data was analysed to assess the prevalence of GBS colonization comparison made according to different stratification e.g. residential area and economic status. Comparison of proportions was made between chosen variables using EPI-INFO version 7 analysis program. The Yates and Chi-squared test and the Kruskal-Wallis $\mathrm{H}$ test were used to compare proportions and medians respectively.

\subsection{Ethical Considerations}

Written informed consent was sought and granted from all mothers recruited into the study. Participants' identity remained anonymous and confidentiality was maintained. Permission to conduct the study was sought and granted from the relevant authorities including the Ethics Committees of Sefako Makgatho Health sciences University and the DGMH management.

\section{Results}

The study recruited 340 pregnant women and GBS colonization was found in $164(48.2 \%)$ participants. Tables 1 and 2 show the socio-demographic features of the women in the study. The characteristics analyzed included age, parity, level of education, marital and employment status. The age range was 18-45 years, with a mean of 30.3 years and Standard Deviation of 6.2 years. The majority of the women were aged between 20 and 40 years. Figure 1 shows the distribution of the proportion of women at different parities. The majority of the women were categorized under Para 1-2, with the Para 5 or more being the least.

Table 1. Demographic features of the pregnant women in the study $[N=$ 340].

\begin{tabular}{ll}
\hline Age range (years) & No. $(\%)$ \\
\hline$<20$ & 92.6 \\
$20-29$ & 15445.3 \\
$30-39$ & 15345.0 \\
$\geq 40$ & 247.1 \\
Total & 340100 \\
\hline
\end{tabular}

In terms of socio-demographic characteristics, the majority of the women recruited were single, had reached matric level of education and were unemployed as shown in Table 2.

Table 2. The distribution of women based on socio-demographic characteristics.

\begin{tabular}{llll}
\hline Socio-demographic characteristics & Variable & No. & $\mathbf{( \% )}$ \\
\hline Marital status & Single & 187 & 55.0 \\
& Married & 89 & 26.2 \\
& Cohabiting & 62 & 18.2 \\
& Divorced & 2 & 0.6 \\
& Below matric & 92 & 27.0 \\
& Matric & 189 & 55.6 \\
& Tertiary & 59 & 17.4 \\
Employment status & Unemployed & 206 & 60.6 \\
& Employed & 134 & 39.4 \\
\hline
\end{tabular}

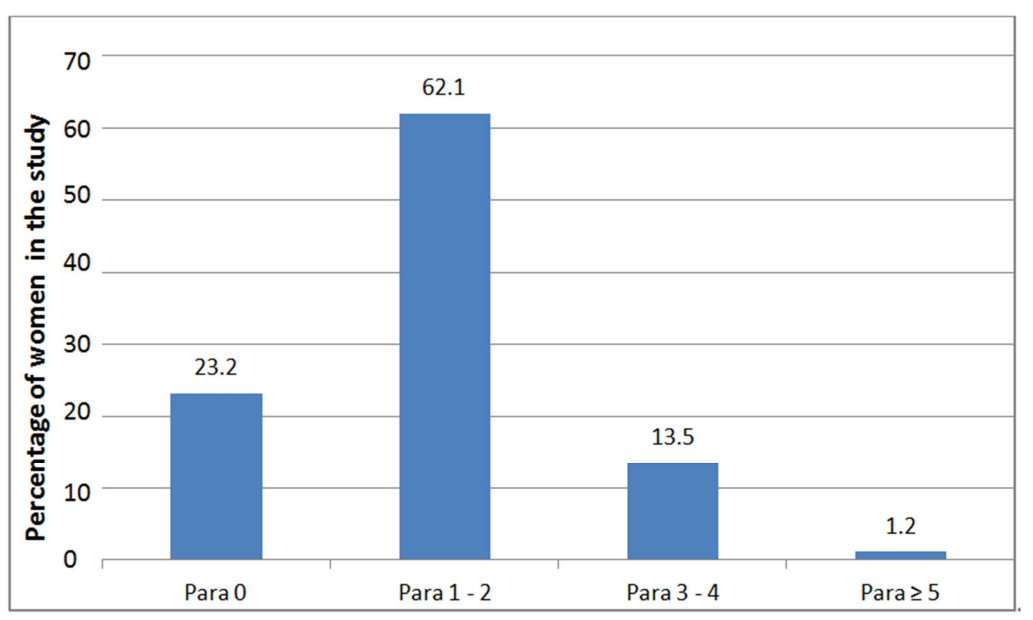

Figure 1. Distribution of women at different parity $(N=340)$.

Table 3 shows the association between GBS colonization and socio-demographic characteristics. It illustrates that the frequency of GBS colonization was significantly associated with being unemployed and having reached Matric level of education. 
Table 3. Association between GBS colonization and socio-demographic characteristics.

\begin{tabular}{|c|c|c|}
\hline & $\begin{array}{l}\text { No. }(\%) \\
\text { GBS }^{+v e}[N=164]\end{array}$ & $\begin{array}{l}\text { No. }(\%) \\
\text { GBS }^{-v e}[N=176]\end{array}$ \\
\hline \multicolumn{3}{|l|}{ Education and GBS status } \\
\hline Below matric & $48(29.3)$ & $44(25.0)$ \\
\hline Matric & $91(55.5)$ & $98(55.7)$ \\
\hline Tertiary & $25(15.2)$ & $\begin{array}{l}34(19.3) \\
{[p<0.0001]}\end{array}$ \\
\hline \multicolumn{3}{|l|}{ Employment and GBS status } \\
\hline Unemployed & $90(54.9)$ & $116(65.9)$ \\
\hline Employed & $74(45.1)$ & $\begin{array}{l}60(34.1) \\
{[p<0.0001]}\end{array}$ \\
\hline
\end{tabular}

Table 4 shows that having no previous miscarriages, stillbirths and absence of a vaginal discharge were associated with GBS colonization.

Table 4. Previous miscarriages, stillbirths and their impact on GBS positivity in the index pregnancy.

\begin{tabular}{llll}
\hline $\begin{array}{l}\text { Obstetric } \\
\text { characteristics }\end{array}$ & Variable & $\begin{array}{l}\text { No. }(\%) \\
\text { GBS }^{+\mathbf{v e}}\end{array}$ & No. (\%) GBS \\
\hline Previous miscarriage & No miscarriage & $126(76.8)$ & $142(80.7)$ \\
& Miscarriage once & $30(18.3)$ & $21(11.9)$ \\
& Miscarriage twice & $7(4.3)$ & $9(5.1)$ \\
& Miscarriage thrice & $1(0.6)$ & $4(2.3)$ \\
& & & {$[p<0.0001]$} \\
Previous stillbirths & No stillbirth & $150(91.5)$ & $166(94.3)$ \\
& Stillbirth once & $12(7.3)$ & $8(4.5)$ \\
& Stillbirth twice & $1(0.6)$ & $2(1.1)$ \\
& Stillbirth thrice/more & $1(0.6)$ & $0(0.0)$ \\
& & & {$[p<0.0001]$} \\
Vaginal discharge & Yes & $27(16.5)$ & $25(14.2)$ \\
& No & $137(83.5)$ & $151(85.8)$ \\
& & & {$[p<0.0001]$} \\
\hline
\end{tabular}

Table 5 shows the profile of comorbidities amongst the women included the study. Most women (91.3\%) did not have any comorbidities. Those who independently had hypertension or asthma comprised $3.2 \%$.

Table 5. Frequency of women with different comorbidities.

\begin{tabular}{lll}
\hline Co-morbidity: & No. & \% \\
\hline No co-morbidity & 312 & 91.3 \\
Diabetes mellitus & 3 & 0.9 \\
Hypertension & 11 & 3.2 \\
Epilepsy & 1 & 0.3 \\
Asthma & 11 & 3.2 \\
Two co-morbidities & 2 & 0.6 \\
Total & 340 & \\
\hline
\end{tabular}

From a total of 340 women $38.2 \%$ were found to be HIV positive. Among those who were colonized with GBS, $41.5 \%$ of them were HIV positive. GBS colonization was significantly associated with HIV positivity $(\mathrm{p}<0.0001)$. Figure 2 shows the distribution of the women based on different duration of the rupture of their membranes. The proportion of women who had a duration of the rupture of $\geq$ 5.0 hours was $36.0 \%$. The range for the duration of rupture of membranes was $0.5-72$ hours and the median was 2 hours. The number of women who had PROM was 8 (4.9\%).

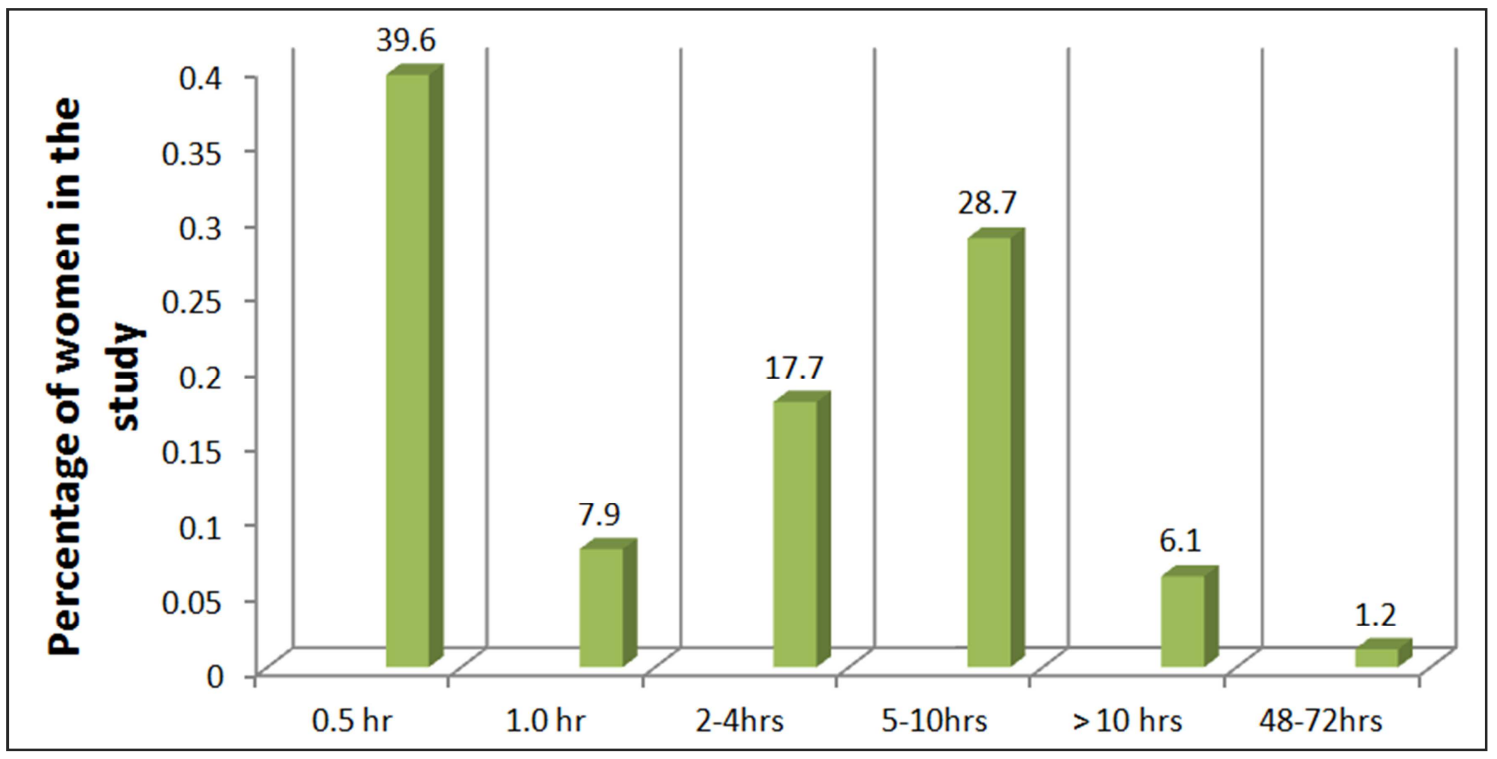

Figure 2. Distribution of women with different duration of the rupture of membranes.

The proportion of women who gave normal vaginal delivery was $54.9 \%$ compared to $45.1 \%$ who delivered by caesarean section. The deliveries which were preterm were $6.1 \%$ compared to $93.9 \%$ full term deliveries. Three women out of the $164(1.8 \%)$ who were positive for GBS had wound sepsis, and one woman $(0.6 \%)$ was found with evidence of endometritis.
Table 6 shows results of the eventual foetal and neonatal outcomes. A total of 170 babies were delivered with six set of twins. Swabs were taken from 29 babies of which 7 (24.1\%) tested positive for GBS. No stillbirths were recorded but a single miscarriage was noted. 
Table 6. Fetal and neonatal outcomes from GBS positive women deliveries.

\begin{tabular}{llll}
\hline & & No. & $(\mathbf{\% )}$ \\
\hline Fetal outcomes & Live & 167 & 98.2 \\
& Stillbirths & 0 & 0.0 \\
Neonatal deaths & & 2 & 1.2 \\
Neonatal sepsis & & 3 & 1.8 \\
Miscarriage & & 1 & 0.6 \\
\hline
\end{tabular}

\section{Discussion}

The prevalence of GBS in the current study was found to be quite high at $48.2 \%$. The prevalence from other studies from the Southern African region ranges from $2 \%$ to $47 \%$ [14, 15]. This was surprisingly a high rate of GBS colonization but was close to a previously recorded high GBS colonization rate reported from two international studies of approximately $54 \%$ $[16,17]$. This was probably due to adequate culture methods used which included use of enrichment and selective media and multiples site sampling. The high rate reflected the true proportion of women who were colonized as it is known that healthy individuals are often colonized especially those with underlying conditions. Many women recruited were HIV positive. An early previously reported average for sub-Saharan Africa has been reported as 19\% implying that there has been an increase over time [18]. A high rate of GBS colonization is often obtained if women are followed up for a certain period and both the rectal and vaginal sites are investigated which was the case in the current study. GBS colonization rates are known to vary with different geographical localities and different communities $[2,16]$. This is attributed to variation in socio-economic factors and sampling techniques. Cultural, ethnic and genetic factors play a role in the variation of the rates of infection with GBS [2, 19, 20]. Lower rates are attributed to false negative culture due to inadequate swabbing technique or poor handling, specimen storage conditions, and prolonged transport. Different positive culture rates are also attributed to use of different culture media [21, 22].

A previous study identified the age less than 20 years as a risk factor [8]. In the current study high GBS colonization rate was associated with an age range of between 20-29 years similar to a previous study [23]. This is a sexually active group and it is known that anogenital colonization by GBS is sexually transmitted $[24,25]$. GBS infection in the current study decreased with increasing age which correlated with a previous study [9]. In that study the age of less than 20 years was not identified as a risk factor. Other studies have not found any association between GBS colonization and maternal age [20]. Gestational age did not influence the rates of maternal colonization with GBS among HIV-1-infected women [26]. GBS colonization was found associated with age, occupation, number of antenatal clinic visits, and gravida [3]. In contrast there were no significant differences between colonized and GBSnegative women with regard to age, marital status, previous miscarriages or abortions, gestational age at examination or at delivery, rate of prematurity, infant's birth weight, or mode of delivery [20].
Several studies have analysed GBS correlation with parity but a clear relationship has not been established as some have been contradictory. In the current study, no significant difference was found with regards to parity between GBS colonized and non-colonized. Several studies [27-29] found no significant differences in colonization rates by age or parity, while others report associations with young age and lower parity [10, 30, 31].

Poor socio-economic status is often implicated as one of the risk factors for GBS colonization. Socio-economic status can be reflected indirectly by level of education and employment status [9]. In the present study lack of education was identified as a risk factor for GBS colonization whereas unemployment was not. This finding is in contrast with other studies in which GBS colonization has been found more frequently among women of low socio-economic class $[6,20$, 32]. In other reports GBS prevalence is reported to be higher among those with lower socio-economic status [33] and lower education [34], but others report higher colonization rates among women who are socially advantaged with a higher education [35].

GBS has been implicated in stillbirths and miscarriages [7, 8]. In the current study previous history of miscarriages and stillbirths in colonized women were not identified as risk factors. This finding correlates with a previous study where the mother's age, educational level, history of pregnancy, maternal complications, and previous neonatal sepsis were not related to GBS colonization [36]. Early neonatal death and gestational age did not have any significant association with GBS colonization in a recent study [23]. There was an association between $S$. agalactiae colonization with preterm labour and PROM in this study. GBS colonization was significantly associated with age group, education, frequency of pregnancy, and PROM [35]. In a different study, GBS positivity was associated with preterm labor. Vaginal colonization with GBS was not associated with preterm labor and PROM [37]. Despite the high colonization rate found in the current study, a few women presented with PROM at $4.9 \%$ with $6.1 \%$ having preterm deliveries. All women with PROM received antibiotics. Caesarean sections were done in 74 (45.1\%) of the women and post-delivery prophylactic antibiotics were given to all. No antibiotics were given to women with normal vaginal deliveries either intrapartum or postpartum. Majority of the women who delivered by caesarean section were electives, because they had a previously scared uterus.

Multiparity was associated with a lower colonization rate. Women of a lower socioeconomic class were associated with GBS colonization. No association was found between colonization and maternal age, previous obstetric history, marital status, nationality, prematurity, caesarean section, or infant birth weight [20].

Twenty (19.8\%) HIV-1-infected pregnant women were found to have GBS colonization between 35 and 37 weeks of gestation [38]. In Malawi the correlation of GBS and HIV was analysed but, no association was found between HIV status and GBS carriage [13]. In the present study HIV positive GBS colonized women were higher than HIV 
positive GBS non-colonized women. In this instance, HIV seems to be a risk factor for GBS colonization. HIV-1 infection was not a risk factor for GBS colonization among an ethnically diverse pregnant population, although data suggested that among HIV-infected women, plasma HIV-1 viremia may be associated with GBS colonization. HIV serostatus was not independently associated with GBS colonization [39]. In contrast carriage was increased among HIV-positive women with higher CD4 counts [13]. No significant increase in GBS colonization was observed in HIV-1-infected pregnant women. Maternal colonization of GBS in HIV-infected pregnant women was not found to be associated with their immunological status [26]. There was no difference in GBS colonization rate between HIV-1 positive and HIV-1 negative pregnant women [26]. Mavenyengwa et al, 2010 did not find HIV infection to be correlated with GBS colonization [40].

In more than $80 \%$ of cases neonatal GBS infection is acquired during pregnancy and delivery by direct mother to child transmission of the pathogen [41]. Seven (24.1\%) babies tested positive for GBS of whom one presented with early neonatal sepsis and was treated and discharged. Generally neonatal outcome was good in the current study despite no intrapartum antibiotics administration having been done. The vertical transmission rate was slightly higher than the previously reported of $22.5 \%$ [20] but much lower in others of $8.9 \%$ [42]. A total of 170 babies were delivered with six set of twins in the current study. Vertical transmission occurs in 30 to $70 \%$ of neonates whose mothers have an GBS positive culture during pregnancy [43]. The rate of transmission of GBS to newborns of colonized women is approximately $50 \%$, and after that $1-2 \%$ of these newborns develop invasive GBS infection in the first week of life [44]. The overall infant colonization rate was $2.1 \%$ [20]. Although transmission rates range from $29 \%$ to $85 \%$ in most studies, rates as low as $12 \%$ have been reported from countries where low colonization rates have also been found despite the use of adequate microbiological methods $[6,41]$.

There was no evidence in the current study to show GBS adverse effects on pregnancy outcome except a few cases of PROM. This was contrary to a previous study which found no association between $S$. agalactiae colonization and higher rates of PROM [26]. GBS colonization has been reported not to be associated with adverse perinatal outcome [15].

Very few women had comorbidities in the current study although a few were recorded as having hypertension and asthma. Diabetes mellitus has been reported to be associated with GBS colonization although this was not the case in the current study [45, 46]. Most cases where GBS has comorbidities involve non-pregnant adults who already have other long term health problems. This has been attributed to immunosuppression [47].

\section{Conclusion}

Colonization by GBS in pregnant women at DGMH was very high. To prevent GBS infection of neonates, clinicians should be alert to the potentially higher risk of GBS colonization in pregnant women. Significant risk factors identified were history of previous stillbirths and miscarriages and lack of education. HIV status had a significant correlation with GBS carriage. Despite the high colonization of GBS, there was generally good maternal and neonatal outcome.

\section{Authors' Contributions}

SML, JYB, MOC and CMM were the primary researchers who, participated in data collection and conducted data analysis. RTM, SRM, SLL, TCT, MN and SM conceived the study, designed, supervised the work and drafted the manuscript for publication. All authors reviewed the initial manuscript drafts, read and approved the final manuscript.

\section{Acknowledgements}

The authors would like to thank Nursing Sisters Malete, Makhathini, and Professor O. A. Towobola.

\section{References}

[1] Narava S, G Rajaram G, Ramadevi A, Prakash GV, Mackenzie S: Prevention of perinatal group B streptococcal infections: A review with an Indian perspective. Indian J Med Microbiol 2014; (32)1: 6-12.

[2] Woldu ZL, Teklehaimanot GT, Waji ST, Gebremariam MY: The prevalence of Group B Streptococus recto-vaginal colonization and antimicrobial susceptibility pattern in pregnant mothers at two hospitals of Addis Ababa, Ethiopia. Reprod Health 2014; 11: 80.

[3] Arain FR, Al-Bezrah NA, Al-Aali KY: Prevalence of Maternal Genital Tract Colonization by Group B Streptococcus From Western Province, Taif, Saudi Arabia. J Clin Gynecol Obstet 2015; 4(3): 258-264.

[4] Dangor Z, Lala SG, Cutland CL, Koen A, Jose L, Nakwa F, Ramdin T, Fredericks J, Wadula J, Madhi SA: Burden of invasive Group B Streptococcus disease and early neurological sequelae in South African infants. PloS ONE 2015; 10(4): e0123014.

[5] Suzman AS, Baltimore RS, Fonseca SNS: Prevalence of maternal group B streptococcal colonization and related risk factors in Brazilian population. Braz J Infect Dis 2006; 10(4): $1433-46$.

[6] Edwards MS, Nizet V, Baker CJ. 2006. Group B Streptococcal infections, p 403-464. In Remington JS, Klein JO, Wilson CB, Baker CJ (ed), Infectious diseases of the fetus and newborn infant, 6th ed. Elsevier Saunders, Philadelphia, PA.

[7] Moyo SR, Tswana SA, Nystrom L, Bergstrom S, Blomberg J, Ljungh A: Stillbirth and intrauterine infection, histological chorioamnionitis and microbiological findings. Int J Gynecol Obstet 1996; 54(2): 115-123.

[8] Hoogkamp-Korstanje JAA, Gerards LJ, Cats BP: Maternal carriage and neonatal acquisition of group B streptococci. J Infect Dis 1982; 145(6): 800-803. 
[9] Dzowela T, Komolafe OO, Igbigbi A: Prevalence of group B Streptococcus colonization in antenatal women at the Queen Elisabeth Central Hospital, Blantyre - a preliminary study. Malawi Med J 2005, 17(3): 97-99.

[10] Yow MD, Leeds L J, Thompson PK, Mason EO Jr, Clark DJ, Beachler CW: The natural history of group B streptococcal colonization in the pregnant woman and their offspring colonization studies. Amer J Obstet Gynecol 1980; 137(1): 34-38.

[11] Brodeur BR, Boyer M, Charlebois I, Hamel J, Couture F, Rioux CR, Martin D: Identification of group B streptococcal Sip protein, which elicits cross protective immunity. Infect Imm 2000; 68(10): 5610-5618.

[12] Busetti M, D’Agaro P, Campello C. Group B streptococcus prevalence in pregnant women from North-Eastern Italy: advantages of screening strategy based on direct plating plus broth enrichment. J Clin Pat 2007; 60: 1140-43.

[13] Gray KJ, Kafulafula G, Matemba M, Kamdolozi M, Membe G, French N: Group B Streptococcus and HIV Infection in Pregnant Women, Malawi, 2008-2010. Emerg Infect Dis 2011; 17(10): 1932-1935.

[14] de Steenwinkel FD, Tak HV, Muller AE, Nouwen JL, Oostvogel PM, S. M. Mocumbi SM: Low carriage rate of group B streptococcus in pregnant women in Maputo, Mozambique. Trop Med Int Health 2008; 13:427-9.

[15] Mavenyengwa RT, Afset JE, Schei B, Berg S, Caspersen T, Bergseng H, Moyo SR: Group B Streptococcus (GBS) colonization during pregnancy and maternal-fetal transmission in Zimbabwe. Acta Obstet Gynecol Scand 2010; 89: 250-5.

[16] Hansen SM, Uldbjerg N, Kilian M, Sørensen UB: Dynamics of Streptococcus agalactiae colonization in women during and after pregnancy and in their infants. J Clin Microbiol 2004; 42(1): 83-89.

[17] Hernández TM, Soriano BD: High prevalence of group B Streptococcus colonization in Mexican pregnant women. Ginecol Obstet Mex. 2006, 74(3): 139-43.

[18] Stoll BJ, Schuchat A: Maternal carriage of group B Streptococci in developing countries. Pediatr Infect Dis J 1998; 17(6): 499-503.

[19] American Academy of Pediatrics Committee on Infectious Diseases and Committee on Fetus and Newborn: Revised guidelines for prevention of early onset group B streptococcal (GBS) infection. Pediatrics 1997, 99: 489-497.

[20] Tsolia M, Psoma M, Gavrili S, Petrochilou V, Michalas S, Legakis N, Karpathios T: Group B streptococcus colonization of Greek pregnant women and neonates: prevalence, risk factors and serotypes. Clin Microbiol Infect 2003; 9: 832-838.

[21] Larsen J, Sever J. Group B streptococcus and pregnancy: a review. Am J Obstet Gynecol 2008; 198(4): 440-448.

[22] Shirazi M, Abbariki E, Ali Hafizi A, Shahbazi F, Bandari M, Dastgerdy E: The prevalence of Group B Streptococcus colonization in Iranian pregnant women and its subsequent outcome. Int J Fertil Steril 2014; 7(4): 267-270.

[23] Gebremeskel TK, Zeleke TA, Mihret A, Tikue MD: Prevalence and Antibiotic Susceptibility Pattern of Streptococcus agalactiae Among Pregnant Women at Adigrat Zonal Hospital and Adigrat Health Center, Tigray, Ethiopia. J Gyn Obstet 2015; 3(2): 29-35.
[24] Foxman B, Gillespie BW, Manning SD, Marrs CF: Risk factors for group B streptococcal colonization: potential for different transmission systems by capsular type. Ann Epidemiol 2007; 17: 854-862.

[25] Manning SD, Tallman P, Baker CJ, Gillespie B, Marrs CF, Foxman B: Determinants of co-colonization with group B Streptococcus among heterosexual college couples. Epidemiol 2002; 13: 533-9.

[26] Beitune P, Duarte G, Maffei CML, Quintana SM, Silva AC, Nogueira AA: Group B Streptococcus carriers among HIV-1 infected pregnant women: prevalence and risk factors. Eur J Obs Gynecol Reprod Biol 2006; 128: 54-58.

[27] Baker CJ, Barrett FF, Yow MD: The influence of advancing gestation on group B streptococcal colonization in pregnant women. Am J Obstet Gynecol 1975; 122: 820-823.

[28] Hastings MJ, Easmon CS, Neill J, Bloxham B, Rivers RP: Group B streptococcal colonisation and the outcome of pregnancy. J Infect 1986; 12: 23-29.

[29] Valkenburg-van den Berg AW, Sprij AJ, Oostvogel PM, Mutsaers JA, Renes WB, Rosendaal FR, Dorr PJ. Prevalence of colonisation with group B Streptococci in pregnant women of a multi-ethnic population in The Netherlands. Eur J Obstet Gynecol Reprod Biol 2006; 124: 178-183.

[30] Aali BS, Abdollahi H, Nakhaee N, Davazdahemami Z, Mehdizadeh A: The association of preterm labor with vaginal colonization of group B streptococci. Iranian J Reprod Med 2007; 5(4): 191-194.

[31] Anthony BF, Okada DM, Hobel CJ: Epidemiology of group B Streptococcus: longitudinal observations during pregnancy. J Infect Dis 1978; 137: 524-530.

[32] McKenna DS, Iams JD: Group B streptococcal infections. Semin Perinatol 1998, 22: 267-76.

[33] Grimwood K, Stone PR, Gosling IA, Green R, Darlow BA, Lennon DR, Martin DR: Late antenatal carriage of group B Streptococcus by New Zealand women. Aust N Z J Obstet Gynaecol 2002; 42: 182-186.

[34] Regan JA, Klebanoff MA, Nugent RP: The epidemiology of group B streptococcal colonization in pregnancy. Vaginal Infections and Prematurity Study Group. Obstet Gynecol 1991; 77: 604-610.

[35] Kim EJ Oh KY, Kim MY, Yong Soo Seo, Shin JH, Song YR, Yang JH, Foxman B, Ki M: Risk Factors for Group B Streptococcus Colonization Among Pregnant Women in Korea. Epidemiol Health. 2011; 33: e2011010.

[36] Javanmanesh F, Eshraghi N. Prevalence of positive rectovaginal culture for Group B streptococcus in pregnant women at 35-37 weeks of gestation. Med J Islam Repub Iran. 2013, 27(1): 7-11.

[37] Kubota T: Relationship between maternal group B streptococcal colonization and pregnancy outcome. Obstet Gynecol 1998; 92: 926-930.

[38] Beitune P, Duarte G, Maffei, CML, Kobori FC: Streptococcus agalactiae colonization among HIV-1-infected pregnant women: antimicrobial susceptibility evaluation. J Acq Imm Defic Synd 2007; 44(2):246. 
[39] Shah M, Aziz N, Leva N, Cohan D. Group B Streptococcus colonization by HIV status in pregnant women: prevalence and risk factors. J Womens Health (Larchmt) 2011; 20(11): $1737-41$.

[40] Mavenyengwa RT, Moyo SR, Nordbø SA: Streptococcus agalactiae colonization and correlation with HIV-1 and Hepatitis B seroprevalence in pregnant women in Zimbabwe. Eur. J. Obst. Gyn. Reprod. Biol 2010; 150: 34-8.

[41] Uh Y, Jang IH, Yoon KJ, Lee CH, Kwon JY, Kim MC: Colonization rates and serotypes of group B streptococci isolated from pregnant women in a Korean tertiary hospital. Eur J Clin Microbiol Infect Dis 1997; 16: 753-6.

[42] Madhi SA, Radebe K, Crew-Brown H, Frasch CE, Arakere G, Mokhachane M, Kimura A: High burden of invasive streptococcus agalactiae disease in South Africa. Annals of Tropical Paediatrics 2003, 23: 15-23.

[43] Baker CJ, Barret FF: Transmission of group B streptococci among parturient women and their neonates. J Pediatr 1973; 83: 919-25.
[44] Shet A, Ferrieri P. Neonatal and maternal group B streptococcal infections: A comprehensive review. Indian J Med Res 2004; 120: 141-50.

[45] Ramos E, Gaudier FL, Hearing LR, Del Valle GO, Jenkins S, Briones D: Group B streptococcus colonization in pregnant diabetic women. Obstet Gynecol 1997; 89: 257-60.

[46] Domingo P, Barquet N, Alvarez M, Coll P, Nava J, Garau J: Group B Streptococcal meningitis in adults: Report of twelve cases and review. Clin Infect Dis 1997; 25: 1180-7.

[47] Sunkara B, Bheemreddy S, Lorber B, Lephart PR, Hayakawa K, Sobel JD, Kaye KS, Marchaim D: Group B Streptococcus infections in non-pregnant adults: the role of immunosuppression. Int J Infect Dis 2011; 16(2012) e182e186. 